\title{
DISTANCE TO THE NEAREST INTEGER AND ALGEBRAIC INDEPENDENCE OF CERTAIN REAL NUMBERS ${ }^{1}$
}

\author{
AVIEZRI S. FRAENKEL
}

1. Introduction and results. The problem is examined of how close $\alpha p / q$ lies to an integer for an infinity of rational numbers $p / q$ from a certain class, and, in particular, of how this proximity depends on the real constant $\alpha$. It turns out, roughly speaking, that $\alpha p / q$ is not too close to an integer for most $\alpha$. There exists, however, an uncountable set of $\alpha$ for which $\alpha p / q$ is very close to an integer for an infinity of $p / q$. Certain subsets of this set can be constructed effectively. Some of these subsets consist of algebraically independent numbers which have the power of the continuum.

More specifically, let $\|x\|$ denote the distance of $x$ to the nearest integer. Let $\left\{P_{1}, \cdots, P_{\ell}\right\},\left\{Q_{1}, \cdots, Q_{t}\right\}$ be finite sets of primes and let $c \geqq 1,0 \leqq \mu \leqq 1$. Let

$$
p=p^{*} p^{\prime}, \quad p^{\prime}=P_{1}^{\rho_{1}} \cdots P_{i}^{p_{s}}, \quad q=Q_{1}^{\sigma_{1}} \cdots Q_{t}^{\sigma_{t}},
$$

where $\rho_{1}, \cdots, \rho_{s}, \sigma_{1}, \cdots, \sigma_{t}$ are non-negative integers, and $p^{*}$ is any integer satisfying

$$
0<p^{*} \leqq c p^{\mu} .^{2}
$$

TheOREM I. Let $\delta>0,0 \leqq \mu \leqq 1, a>0$, $\alpha$ real algebraic. Then the inequality $0<\|\alpha p / q\|<q^{(\mu+\delta)}$ holds only for a finite number of $p, q$ of the above form with $p \geqq a q$, provided that $q=1$ if $\mu=1$. In addition, for almost all real $r$-tuples $\left(\alpha_{1}, \cdots, \alpha_{r}\right), r \geqq 1$, the set of inequalities

$$
0<\left\|\alpha_{i} p / q\right\|<p^{-(\mu / r+\delta)}, \quad i=1, \cdots, r,
$$

has only a finite number of solutions in $p, q$ of the above form with $p \geqq a q$, provided that $q=1$ if $\mu=1$.

Presented to the Society, January 25, 1962, under the title Distance to nearest integer of powers of rational numbers; received by the editors November 13, 1961 and, in revised form, August 2, 1963.

1 Part of this work was done while the author was at the University of Oregon.

${ }^{2}$ Since $p=p^{*} p^{\prime}$, this inequality is equivalent to $0<p^{*} \leqq c^{1 /(1-\mu)}\left(p^{\prime}\right)^{\mu /(1-\mu)}$ for $0 \leqq \mu<1$. For $\mu=1$ it becomes vacuous, i.e., $p^{*}$ is unrestricted. 
TheOREM II. Let $\alpha_{1}, \cdots, \alpha_{r}$ be any real numbers, $0 \leqq \mu \leqq 1, K>0$ arbitrary if $0 \leqq \mu<1$ and $K=1$ if $\mu=1$. Let $p^{\prime}, q$ be any integers of the form (1), with the condition that $p^{\prime}=1$ if $\mu=1$. There exists a constant $c>1$ depending only on $r, K, \mu$, the $\alpha_{i}$ and the primes $P_{1}, \cdots, P_{s}$, $Q_{1}, \cdots, Q_{t}$, and an integer $p=p^{*} p^{\prime}$ such that

$$
\left\|\alpha_{i} p / q\right\|<K p^{-\mu / r}, \quad i=1, \cdots, r,
$$

where $0<p^{*} \leqq c p^{\mu}$. Thus (4) has an infinity of solutions in $p, q$ of the form (1) subject to (2), where $p^{\prime}, q$ are arbitrarily prescribed integers of the form (1) subject to the condition $p^{\prime}=1$ if $\mu=1$.

Let $\eta>0, a>0$. For almost all real $r$-tuples $\left(\alpha_{1}, \cdots, \alpha_{r}\right)$, all but $a$ finite number of the $p \geqq a q$, for which (4) holds, satisfy $p^{*}>p^{\mu-\eta}$.

The case $\mu=0$ of the first part of Theorem $I$ is equivalent to a Theorem of Mahler [6, Theorem 1, p. 151], [5]. The conditions $p \geqq a q$ in Theorems I and II are nécessary. Thus, for $s=t=1, P=2$, $Q=5, p^{*}=1$, and for every $0<\delta<\log _{2} 5-2,0 \leqq \mu \leqq 1,0<\alpha<1$, we can write $0<\|\alpha p / q\|=\left\|\alpha(2 / 5)^{n}\right\|<2^{-n(\mu+\delta)}<2^{-n \mu}$ for all $n \geqq 1$. The first part of Theorem II shows that Theorem I is best possible. The second part shows that the leeway for $p^{*}$ indicated by (2) is not usually realized, i.e., the $p^{*}$ satisfying (4), for which $p \geqq a q$, are usually close to their upper bound.

Let $P$ be a prime, $c_{0}>1$. For any $\mu$ in $[0,1)$, let $\left\{p_{1}^{*}, p_{2}^{*}, \cdots\right\}$ be a sequence of integers such that for some index $N=N(\mu), p_{N}^{*}=r_{N}$, $p_{i}^{*}=r_{i} r_{i-1} \cdots r_{N}, i>N$, where the $r_{i}$ are positive integers not divisible by $P, p_{i}^{\mu} \leqq p_{i}^{*} \leqq c_{0} p_{i}^{\mu}, p_{i}=p_{i}^{*} p_{i}^{\prime}, i \geqq N$, and $p_{i}^{*}=1$ for $1 \leqq i<N$. We are interested in these sequences for $p_{i}^{\prime}=P^{i l}$ and $p_{i}^{\prime}=P^{2^{i}}$, and denote the sequences by $S_{\mu}$ and $T_{\mu}$ for these two cases, respectively. Further, let $U=\left\{p_{1}^{*}, p_{2}^{*}, \cdots\right\}$ be a sequence of integers such that $p_{1}^{*}=r_{1}$, $p_{i}^{*}=r_{i} r_{i-1} \cdots r_{1}, i \geqq 1$, where the $r_{i}$ are positive integers not divisible by $P, P^{2 i}<p_{i}^{*}<P^{2(i+1)}$. It is easily seen that such sequences exist. In fact, we can take $S_{0}=T_{0}=\{1,1, \cdots\}$; if $\mu>0$, it suffices if $N$ is sufficiently large to satisfy $\left(p_{N}^{\prime}\right)^{\mu /(1-\mu)}\left(1-c_{0}^{-1 /(1-\mu)}\right)>2$ for both $S_{\mu}$ and $T_{\mu}$. For $\mu>0$ it can even be arranged that all $r_{i}(i>N)$ in $S_{\mu}$ and $T_{\mu}$ be distinct primes $\neq P$. In this case $N$ must, in addition, be so large that the interval

$$
\left(\left(p_{i}^{\prime}\right)^{\mu /(1-\mu)}\left(p_{i-1}^{*}\right)^{-1}, c_{0}^{1 /(1-\mu)}\left(p_{i}^{\prime}\right)^{\mu /(1-\mu)}\left(p_{i-1}^{*}\right)^{-1}\right)
$$

contains a prime $>P$ for all $i \geqq N$, and that these intervals be disjoint for all $i \geqq N$. Using these sequences, a real number can be constructed for which (3) has an infinity of solutions in integers $p, q$ of the form (1) subject to (2). But first we show that there are not too many such numbers. 
Theorem III. Let $\delta>0, a>0$. For fixed $0 \leqq \mu \leqq 1$, let $R(\mu)$ be the set of all real r-tuples $\left(\alpha_{1}, \cdots, \alpha_{r}\right)$ for which (3) has an infinity of solutions in $p, q$ of the form (1) subject to (2) with $p \geqq a q$. Let $R=U_{0 \leq \mu \leq 1} R(\mu)$. Then $R$ has measure 0.

Theorem IV. Let $0<\delta<1-\mu, c>1$. For each $0 \leqq \mu<1$ there exists an everywhere dense subset of Liouville numbers $\alpha$ for which $\|\alpha p / q\|$ $<p^{-(\mu+\delta)}$ has an infinity of solutions in $p, q$ of the form (1) subject to

$$
p^{\mu} \leqq p^{*} \leqq c p^{\mu}
$$

with $q \rightarrow \infty$. For $q=1$ there are Liouville numbers $\alpha_{k}=\sum_{i=1}^{\infty}\left(p_{i r}^{*} P^{(i k) !}\right)^{-1}$, $p_{i k}^{*} \in S_{\mu}, k=1, \cdots, r, 0 \leqq \mu<1$ and non-Liouville transcendental numbers $\alpha_{k}=\sum_{i=1}^{\infty}\left(p_{i k}^{*} P^{2^{i k}}\right)^{-1}, \quad p_{i k}^{*} \in T_{\mu}, k=1, \cdots, r, \quad 0 \leqq \mu<1$, which satisfy $\left\|\alpha_{k} p\right\|<p^{-(\mu / r+\delta)}, k=1, \cdots, r$ for an infinity of $p$ of the form (1) subject to (5).

Theorem V. Let $0 \leqq \mu<1, \delta>0$. Each of the following four sets is algebraically independent over the rationals and, for any number $\xi$ of these sets, $\|\xi p\|<p^{-(\mu+\delta)}$ for an infinity of $p$ of the form (1) subject to (5):

1. The Liouville numbers $\alpha_{k}, k=1,2, \cdots$, defined in Theorem IV.

2. $\quad \beta_{k}=\sum_{i=1}^{\infty}\left(p_{i k}^{*} P^{k(i k) !}\right)^{-1}, p_{i k}^{*} \in S_{1 / 2}, \quad k=1,2, \cdots$

3. $\alpha(x)=\sum_{i=1}^{\infty} p(x, i)^{-1}=\sum_{i=1}^{\infty}\left(p^{*}(x, i) P^{\left[i^{i+x}\right]}\right)^{-1}, \quad p^{*}(x, i) \in U$, $p(x, i)^{x} \leqq p^{*}(x, i) \leqq P^{4} p(x, i)^{x}, 0<x<1$.

4. $\beta(x, y)=\sum_{i=1}^{\infty} p(x, y, i)^{-1}=\sum_{i=1}^{\infty}\left(p^{*}(x, y, i) P^{\left[i^{(i+x)^{i+y}}\right]}\right)^{-1}$,

$$
\begin{aligned}
& p^{*}(x, y, i) \in U, p(x, y, i)^{x} \leqq p^{*}(x, y, i) \leqq P^{4} p(x, y, i)^{*}, \\
& 0<x<1,0<y<1 .
\end{aligned}
$$

From Theorem IV there follows, in particular, that for each $\alpha$ for which (3) (with $r=1$ ) has only a finite number of solutions, there exists a sequence $\{\beta\}$ of real numbers converging to $\alpha$, such that (3) has an infinity of solutions $p, q$ of the specified form for every $\delta>0$ if $\alpha$ is replaced by $\beta$.

The sequence $\left\{\alpha_{k}\right\}$ considered in Theorem $\mathrm{V}$ constitutes an independent set for each (fixed) $\mu$ in $[0,1)$. The case $\mu=0$ is the case usually considered [3], [4], [7], [9]. On the other hand, $\mu$ is not fixed in the sequence $\left\{\beta_{k}\right\}$. The number $\beta_{k}$ is associated with the value $\mu=(1+k)^{-1}$. Indeed, $p_{i k}^{*} \in S_{1 / 2}$ implies $P^{(i k) !} \leqq p_{i k}^{*} \leqq c_{0}^{2} P^{(i k) !}$. Choosing $c_{0}$ to satisfy $1<c_{0}^{2} \leqq c$ and letting $\mu=(1+k)^{-1}$, we have $P^{k(i k) ! \mu /(1-\mu)}$ 
$\leqq p_{i k}^{*} \leqq c^{1 /(1-\mu)} p^{k(i k) ! \mu /(1-\mu)}$, which is equivalent to (5). The numbers $\alpha(x)$ and $\beta(x, y)$ each form a set with the power of the continuum. For fixed $y$, the numbers $\beta(x, y)$, form a subset with the power of the continuum, each number of which corresponds to some value $\mu=x$ in $(0,1)$. For fixed $x$ they also form a subset with the power of the continuum.

2. Proofs. We require a Theorem of Ridout [8]: Let $\alpha$ be algebraic $\neq 0$. Let $\left\{P_{1}, \cdots, P_{3}\right\},\left\{Q_{1}, \cdots, Q_{t}\right\}$ be finite sets of primes, $\mu, \nu, \delta, c$ real numbers satisfying $0 \leqq \mu \leqq 1,0 \leqq \nu \leqq 1, \delta>0, c \geqq 1$. Let $p, q$ be restricted to integers of the form

$$
\begin{aligned}
p & =p^{*} p^{\prime}, & q & =q^{*} q^{\prime}, \\
p^{\prime} & =P_{1}^{p_{1}} \cdots p_{\bullet}^{p_{t}}, & q^{\prime} & =Q_{1}^{\sigma_{1}} \cdots Q_{t}^{\sigma_{t}},
\end{aligned}
$$

where $\rho_{1}, \cdots, \rho_{2}, \sigma_{1}, \cdots, \sigma_{t}$ are non-negative integers, and $p^{*}, q^{*}$ are any integers satisfying $\left|p^{*}\right| \leqq c|p|^{\mu}, 0<q^{*} \leqq c q^{*}$. Then the inequality

$$
0<|\alpha-p / q|<q^{-(\mu+\nu+\delta)}
$$

has only a finite number of solutions in $p, q$ of the above form.

Proof of Theorem I. Suppose that for some $0 \leqq \mu \leqq 1, \delta>0$, there exists an $r$-tuple $\left(\alpha_{1}, \cdots, \alpha_{r}\right)$ for which (3) has an infinity of solutions of the specified form with $p \geqq a q$. Without loss of generality we may assume that $\alpha_{i}$ is positive, $i=1, \cdots, r$. With each solution associate a value $\theta=\theta(p)$ defined by $p^{*}=p^{\theta} \leqq c p^{\mu}$. The infinite sequence $\{\theta(p)\}$ lies in $[0, \mu]$. Let $\zeta=\lim \sup (\theta(p))($ as $p \rightarrow \infty)$. Then for every $\epsilon>0$ there exists an infinity of $p, q$ of the form (1) subject to

$$
p^{5-\bullet}<p^{*} \leqq p^{5}
$$

satisfying (3). For each of these solutions define $\lambda=\lambda(p)$ by $\left(p^{\prime}\right)^{1-\lambda}$ $=q^{1-\zeta}$, where we let $\lambda=1$ if $p^{\prime}=1$. Thus $\lambda \leqq 1$. Let $\nu=\lim \sup (\lambda(p))$ (as $p \rightarrow \infty)$. Then $\nu=1$ if $\zeta=1$. If $\zeta<1$,

$$
\left(p^{\prime}\right)^{(1-\lambda) /(1-\zeta)}=q \leqq a^{-1} p \leqq a^{-1}\left(p^{\prime}\right)^{1 /(1-\zeta)}
$$

by the right-hand side of (6). Hence $\left(p^{\prime}\right)^{\lambda} \geqq a^{1-\zeta}$, and for any $\eta>0$, $\lambda>-\eta$ for all sufficiently large $p^{\prime}$. Hence $0 \leqq \nu \leqq 1$, and there exists an infinity of $p, q$ of the form (1) subject to (6) and

$$
\left(p^{\prime}\right)^{1-\gamma} \leqq q^{1-\zeta}<\left(p^{\prime}\right)^{1-v+e},
$$

satisfying $0<\left|\alpha_{i} p / q-u_{i}\right|<p^{-(\zeta / r+\delta)}, i=1, \cdots, r$, where $u_{i}$ is the nearest integer to $\alpha_{i} p / q$.

Let $l_{i}=u_{i} q$. If $\nu<1$ (and hence $\zeta<1$ ), then $u_{i}<\alpha_{i} p q^{-1}+1$ $\leqq \alpha_{i}\left(p^{\prime}\right)^{1 /(1-r)} q^{-1}+1 \leqq \alpha_{i} q^{\nu /(1-r)}+1 \leqq c_{1} q^{\nu /(1-r)}$, by the right-hand side of 
(6) and the left-hand side of (7), where $c_{1}=1+\max _{i} \alpha_{i}>1$. Hence $u_{i}<c_{2} l_{i}, c_{2}>1$. But this clearly holds also if $\nu=1$. Now,

$$
0<\left|\alpha_{i}-l_{i} / p\right|<\frac{1}{p^{\zeta} / r+\nu+\delta} \cdot \frac{q}{p^{1-\nu}}, \quad i=1, \cdots, r,
$$

infinitely often. But if $\zeta<1$, then

$$
q<\left(p^{\prime}\right)^{(1-v+c) /(1-\zeta)}<p^{(1-\zeta+\epsilon)(1-v+c) /(1-\zeta)}=p^{1-v+\eta}
$$

by the right-hand side of (7) and the left-hand side of (6), where $\eta>0$ is arbitrarily small with $\epsilon$. If $\zeta=1$, then $\mu=\nu=1$ and $q=1=p^{1-\nu}$. In either case, the inequality

$$
0<\left|\alpha_{i}-l_{i} / p\right|<p^{-(\zeta / r+\nu+\delta-\eta)}
$$

has an infinity of solutions in $l_{i}, p$ of the specified type. Hence by Ridout's Theorem, with $\mu$ replaced by $\nu, \nu$ replaced by $\zeta, \delta$ by $\delta-\eta>0$, $c$ by $c_{2}, p$ by $l$ and $q$ by $p, \alpha$ must be transcendental if $r=1$. Since for $r \geqq 1$ Ridout's Theorem is true for almost all real $r$-tuples [ 1 ; Theorem II, p. 85], $\left(\alpha_{1}, \cdots, \alpha_{r}\right)$ must in fact belong to a set of measure $0 .^{3}$

Proof of Theorem II. Assume first $0 \leqq \mu<1$. Let $k \geqq 1, p^{\prime}, q$ any integers of the form (1), $A=\left[2 k\left(p^{\prime}\right)^{\mu / r(1-\mu)}\right]$. As in [1, Lemma 4, pp. 92-93], divide the $r$-dimensional unit cube into $A^{r}$ boxes, and apply Dirichlet's box principle to conclude existence of $x_{1}, x_{2}$ with $0 \leqq x_{1}<x_{2}$ $\leqq A^{r}$ satisfying

$$
\begin{array}{r}
\left|\left(x_{2}-x_{1}\right) \alpha_{i} p^{\prime} / q-\left(\left[x_{2} \alpha_{i} p^{\prime} / q\right]-\left[x_{1} \alpha_{i} p^{\prime} / q\right]\right)\right|<1 / k\left(p^{\prime}\right)^{\mu / r(1-\mu)}, \\
i=1, \cdots, r .
\end{array}
$$

Let $p^{*}=x_{2}-x_{1}$. Then for $c=(2 k)^{r(1-\mu)}$, we have $0<p^{*}$ $\leqq c^{1 /(1-\mu)}\left(p^{\prime}\right)^{\mu /(1-\mu)}$. Letting $u_{i}=\left[x_{2} \alpha_{i} p^{\prime} / q\right]-\left[x_{1} \alpha_{i} p^{\prime} / q\right]$, we obtain

$$
\begin{aligned}
\left\|\alpha_{i} p / q\right\| & \leqq\left|\alpha_{i} p / q-u_{i}\right|<1 / k\left(p^{\prime}\right)^{\mu / r(1-\mu)} \leqq c^{\mu / r(1-\mu)} / k p^{\mu / r} \\
& =2^{\mu} / k^{1-\mu} p^{\mu / r}<K p^{-\mu / r}, \quad i=1, \cdots, r,
\end{aligned}
$$

for all sufficiently large $k$.

If $\mu=1$, apply the box principle with $A$ replaced by $k \geqq 1$. Then

$$
\left|\left(x_{2}-x_{1}\right) \alpha_{i} / q-\left(\left[x_{2} \alpha_{i} / q\right]-\left[x_{1} \alpha_{i} / q\right]\right)\right|<k^{-1}, \quad i=1, \cdots, r .
$$

Added in proof. If (6) does not hold, then $p^{5}<p^{*} \leqq p^{5-6}$ must hold. In this case we define $\lambda$ by $\left(p^{\prime}\right)^{1-\lambda}=q^{1-\zeta-6}$, and the exponent of $q$ in $(7)$ becomes $1-\zeta-\epsilon$. Similarly, the $\epsilon$ in (7) may have to be put on the left side, in which case the remainder of the proof is again modified slightly. 
Letting $p^{*}=p=x_{2}-x_{1} \leqq k^{r}, u_{i}=\left[x_{2} \alpha_{i} / q\right]-\left[x_{1} \alpha_{i} / q\right]$, we have

$$
\left\|\alpha_{i} p / q\right\| \leqq\left|\alpha_{i} p / q-u_{i}\right|<k^{-1} \leqq p^{-1 / r} \text {. }
$$

Suppose that the second part of the theorem is false. This means that there exist $\eta>0, a>0$ and a set of $r$-tuples $\left(\alpha_{1}, \cdots, \alpha_{r}\right)$ of positive measure, for which (4) has an infinity of solutions in $p, q$ of the form (1) subject to $p^{*} \leqq p^{\mu-\eta}, p \geqq a q$. Replacing $\mu-\eta$ by $\mu$, we have $p^{*} \leqq p^{\mu}$ and $\left\|\alpha_{i} p / q\right\|<K p^{-(\mu / r+c),} i=1, \cdots, r$, infinitely of ten for any $0<\epsilon \leqq \eta / r$, and for a set of $r$-tuples of positive measure, contradicting Theorem $\mathrm{I}$.

Proof of Theorems III, IV and V. The proof of Theorem III is based on the second part of Theorem I. Its proof is very similar to the proof of [2, Theorem IV], and is therefore omitted. The proof of the first part of Theorem IV follows directly from [2, Theorem I]. For proving the second part, concerning the non-Liouville numbers, let

$$
\frac{l_{m, k}}{p_{m, k}}=\sum_{i=1}^{m r ! / k}\left(p_{i k}^{*} P^{2^{i k}}\right)^{-1}, \quad k=1, \cdots, r,
$$

where $m$ is any positive integer. Then $p_{m, k}=p_{m r 1}^{*} P^{2^{m r 1}}$ for $k=1, \cdots, r$, and

$$
\left.\alpha_{k}-\frac{l_{m, k}}{p_{m, k}}=\sum_{D m r 1 / k}\left(p_{i k}^{*} P^{2^{i k}}\right)^{-1}<2\left(p_{m r 1+k}^{*} P^{2 r_{r l+k}}\right)\right)^{-1} .
$$

But $2 P^{-2^{m+1+k}}<\left(P^{-2^{m+1}}\right)^{1+\mu+\delta}, p_{m r l+k}^{*-1} \leqq p_{m+1}^{*-(1+\mu+\delta)}$, if $m$ is sufficiently large and $0<\delta<1-\mu$. Hence $\left\|\alpha_{k} p\right\|<p^{-(\mu+\delta)} \leqq p^{-(\mu / r+\delta)}, k=1, \cdots, r$ for an infinity of $p_{m, k}=p$, and $\alpha_{k}$ is transcendental by Ridout's Theorem. (Since the first terms of the series defining $\alpha_{k}$ can be chosen at will, the numbers $\alpha_{k}$ are also everywhere dense.) The same proof holds for the Liouville numbers.

For proving Theorem V, we use Schmidt's independence criterion for Liouville numbers [9]: Let $\left(\alpha_{1}, \cdots, \alpha_{n}\right)$ be an $n$-tuple of reals such that for every $d>0$ there exists an integer $p$ with

$$
0<\left\|\alpha_{k} p\right\|<\left(\left\|\alpha_{1} p\right\| \cdots\left\|\alpha_{k-1} p\right\|\right)^{d} p^{1-n d}, \quad k=1, \cdots, n .
$$

Then $\alpha_{1}, \cdots, \alpha_{n}$ are algebraically independent over the rationals. To show that the $\beta(x, y)$ are independent, it suffices to apply Schmidt's criterion to the $n^{2}$-tuple $\left(\beta\left(x_{0}, y_{0}\right), \beta\left(x_{1}, y_{0}\right), \cdots, \beta\left(x_{n-1}, y_{0}\right), \beta\left(x_{0}, y_{1}\right)\right.$, $\left.\cdots, \beta\left(x_{n-1}, y_{n-1}\right)\right)$, where $n$ is fixed and $0<x_{0}<\cdots<x_{n-1}<1$, $0<y_{0}<\cdots<y_{n-1}<1$. Let

$$
p_{h}=p^{*}(h) P^{h^{h^{h}}}, p^{*}(h) \in U, p^{h^{{ }^{h}}} \leqq p^{*}(h) \leqq P^{1+h^{h^{h}}} .
$$


If $h$ is sufficiently large,

$$
\left\|\beta(x, y) p_{h}\right\|=p_{h} \sum_{i=0}^{\infty}\left(p^{*}(x, y, h+i) P^{\left[(h+i)^{(h+i+x)^{h+i+y}}\right]}\right)^{-1} .
$$

Hence, letting $f_{x, y}=p^{*}(h) / p^{*}(x, y, h)$,

$$
f_{x, y} P^{h^{h^{h}}-\left[{ }_{h}(h+x)^{h+y}\right]}<\|\beta(x, y)\|<2 f_{x, y} P^{h^{h^{h}}-\left[{ }_{h}(h+x)^{h+y}\right] .}
$$

If $k-1=v n+r, 0 \leqq r<n$, then

$$
\Pi\left\|\beta\left(x_{i}, y_{j}\right)\right\|^{d} p_{h}^{1-n d}>p^{*}(h) p^{*}\left(x_{r}, y_{v}, h\right)^{-n d} P^{h^{h}}-\left[h_{h}^{\left.\left(h+x_{r}\right)^{h+y_{v}}\right]},\right.
$$

where the product is taken over all $i, j$ satisfying $0 \leqq i+j n \leqq k-1$, $0 \leqq i<n$. It is easily verified that

and

$$
P^{h^{h}{ }^{h}{ }_{-n d}\left[\left(h+x_{r}\right)^{h+y_{v}}\right]}> \begin{cases}\left.P^{h^{h}}-\left[h^{\left(h+x_{r}+1\right.}\right)^{h+y_{v}}\right], & \text { if } 0 \leqq r<n-1, \\ P^{h^{h}-[h}{ }^{\left.\left(h+x_{0}\right)^{h+y_{v+1}}\right],} & \text { if } r=n-1,\end{cases}
$$

$$
p(h)^{*} p^{*}\left(x_{r}, y_{v}, h\right)^{-n d}> \begin{cases}f_{x_{r+1}, y_{v},}, & \text { if } 0 \leqq r<n-1, \\ f_{x_{0}, y_{v}+1}, & \text { if } r=n-1,\end{cases}
$$

if $h=h(d)$ is sufficiently large. Hence Schmidt's criterion is satisfied. For the numbers $\alpha_{k}$ the criterion is applied with $p_{h}=p_{h}^{*} P^{n !}, p_{h}^{*} \in S_{\mu}$, $n ! \mid h$, for the $\beta_{k}$ with $p_{h}=p_{n}^{*} P^{n !}, p_{h}^{*} \in S_{1 / 2}, n ! \mid h$, and for the $\alpha(x)$ it is applied with $p_{h}=p^{*}(h) P^{h^{h}}, p^{*}(h) \in U, P^{h^{h}}<p^{*}(h)<P^{4+h^{h}}$.

\section{REFERENCES}

1. A. S. Fraenkel, On a Theorem of D. Ridout in the theory of Diophantine approximations, Trans. Amer. Math. Soc. 105 (1962), 84-101.

2. - Transcendental numbers and a conjecture of Erdös and Mahler, J. London Math Soc. 39 (1964), 405-416.

3. H. Kneser, Eine kontinuumsmächtige, algebraisch unabhängige Menge reeller Zahlen, Bull. Soc. Math. Belg. 12 (1960), 23-27.

4. F. Kuiper and J. Popken, On the so-called von Neumann numbers, Nederl. Akad. Wetensch. Proc. Ser. A 65 (1962), 385-390.

5. K. Mahler, On the fractional parts of the powers of a rational number. II, Mathematika 4 (1957), 122-124.

6. - Lectures on Diophantine approximations, Part 1, University of Notre Dame, Notre Dame, Ind., 1961.

7. J. von Neumann, Ein System algebraisch unabhängiger Zahlen, Math. Ann. 99 (1928), 134-141.

8. D. Ridout, Rational approximations to algebraic numbers, Mathematika 4 (1957), 125-131.

9. W. M. Schmidt, Simultaneous approximation and algebraic independence of numbers, Bull. Amer. Math. Soc. 68 (1962), 475-478.

The Weizacann Institute of Science, Rehovot, Israel 